\title{
AN OUTFLOW PERPENDICULAR TO THE RADIO JET IN THE SEYFERT NUCLEUS OF NGC 5929
}

\author{
Rogemar A. Riffel ${ }^{1}$, Thaisa Storchi-BergmanN ${ }^{2}$, AND Rogério RifFeL ${ }^{2}$ \\ ${ }^{1}$ Departamento de Física, CCNE, Universidade Federal de Santa Maria, 97105-900 Santa Maria, RS, Brazil; rogemar@ufsm.br \\ ${ }^{2}$ IF, Universidade Federal do Rio Grande do Sul, CP 15051, Porto Alegre 91501-970, RS, Brazil; thaisa@ufrgs.br, riffel@ufrgs.br \\ Received 2013 July 5; accepted 2013 November 22; published 2013 December 16
}

\begin{abstract}
We report the observation of an outflow perpendicular to the radio jet in near-infrared integral field spectra of the inner $250 \mathrm{pc}$ of the Seyfert 2 galaxy NGC 5929. The observations were obtained with the Gemini Near-infrared Integral Field Spectrograph at a spatial resolution of $\sim 20 \mathrm{pc}$ and spectral resolution of $R \approx 5300$ and reveal a region $\sim 50 \mathrm{pc}$ wide crossing the nucleus and extending by $\sim 300 \mathrm{pc}$ perpendicularly to the known radio jet in this galaxy. Along this structure-which we call the south-east-north-west (SE-NW) strip-the emission line profiles show two velocity components, one blueshifted and the other redshifted by $-150 \mathrm{~km} \mathrm{~s}^{-1}$ and $150 \mathrm{~km} \mathrm{~s}^{-1}$, respectively, relative to the systemic velocity. We interpret these two components as being due to an outflow perpendicular to the radio jet, which is supported by low-frequency radio emission observed along the same region. We attribute this feature to the interaction of ambient gas with an "equatorial outflow" predicted in recent accretion disk and torus wind models. Perpendicularly to the SE-NW strip, thus approximately along the radio jet, single-component profiles show blueshifts of $\approx-150 \mathrm{~km} \mathrm{~s}^{-1}$ to the north-east and similar redshifts to the south-west, which can be attributed to gas counter-rotating relative to the stellar kinematics. More double-peaked profiles are observed in association with the two radio hot spots, attributed to interaction of the radio jet with the surrounding gas.
\end{abstract}

Key words: galaxies: active - galaxies: individual (NGC 5929) - galaxies: ISM - galaxies: nuclei

Online-only material: color figures

\section{INTRODUCTION}

Signatures of interactions between radio jets and the narrow line region (NLR) emitting gas in Seyfert galaxies have been frequently reported in the literature and usually appear in the data as distorted emission line profiles relative to a Gaussian. These profiles show, for example, increased velocity dispersions, red or blue wings, and other kinematic signatures attributed to outflows observed in regions co-spatial with radio structures (e.g., Falcke et al. 1998; Cecil et al. 2002; Whittle \& Wilson 2004; Riffel et al. 2006; Riffel \& Storchi-Bergmann 2011). Fast shocks produced by the radio jet can modify the shape of the NLR by compressing the gas and producing line emission enhancement in regions next to radio structures. Usually, the interaction of the radio jet with the NLR is seen only in association with highfrequency radio emission (e.g., from 1.6 to $15 \mathrm{GHz}$ ). On the other hand, outflows observed in Seyfert galaxies are not usually originated by the interaction of the radio jet with the NLR gas, but by the interaction of winds from the accretion disk with gas from the circumnuclear region of the galaxy. These outflows have been observed to have a bi-conical shape in a number of nearby Seyfert galaxies (e.g., Storchi-Bergmann et al. 1992, 2010; Das et al. 2005, 2007).

NGC 5929 is a spiral galaxy harboring a Seyfert 2 nucleus, located at a distance $35.9 \mathrm{Mpc},{ }^{3}$ for which 1 arcsec corresponds to $\sim 175 \mathrm{pc}$ at the galaxy. It presents a well-defined bi-polar radio jet oriented along the position angle P.A. $\approx 60^{\circ}$, showing a triple structure with two bright hot spots, one located at 0 '.5 north-east from the nucleus and the other at 0.' 6 south-west from it. The third and fainter radio structure is observed at the nucleus of the galaxy (Ulvestad \& Wilson 1984; Wilson \& Keel 1989; $\mathrm{Su}$ et al. 1996). Besides this main jet, weak extended emission is observed at $400 \mathrm{MHz}$, perpendicularly to the main radio jet (Su et al. 1996), and approximately along the minor axis of the

\footnotetext{
3 From the NASA/IPAC Extragalactic Database, http://ned.ipac.caltech.edu/.
}

galaxy. NGC 5929 seems to be in interaction with its companion galaxy NGC 5930.

The gas kinematics of the nuclear region of NGC 5929 has been discussed in several previous works, but its origin is still not well understood (Keel 1985; Ferruit et al. 1999; Rosario et al. 2010).

In this Letter, we present two-dimensional kinematic maps of the inner $250 \mathrm{pc}$ (radius) of NGC 5929 and report the observation of double-component emission at the location of the radio hot spots, but also along a $50 \mathrm{pc}$ wide strip perpendicular to the radio jet using near-infrared integral field spectroscopy (IFS) at the Gemini North telescope.

\section{OBSERVATIONS}

NGC 5929 was observed using the Gemini Near-infrared Integral Field Spectrograph (McGregor et al. 2003) operating with the adaptive optics module ALTAIR under the observation program GN-2011A-Q-43. The observations covered the $J$ and $K_{1}$ spectral bands, resulting in a wavelength coverage from $1.14 \mu \mathrm{m}$ to $1.36 \mu \mathrm{m}$ and $2.10 \mu \mathrm{m}$ to $2.54 \mu \mathrm{m}$, respectively. The total on-source exposure time for each band was $6000 \mathrm{~s}$ and the observations were split into ten individual on-source exposures plus five sky exposures for each band. The final data cubes cover the inner $3^{\prime \prime} \times 3^{\prime \prime}$ of NGC 5929 at an angular resolution of 0 "'.12 (corresponding to $\sim 20 \mathrm{pc}$ at the galaxy) and velocity resolution of $\sim 40 \mathrm{~km} \mathrm{~s}^{-1}$.

\section{RESULTS}

In order to construct flux, velocity dispersion $(\sigma)$ and centroid velocity maps for the emitting gas ([PII], [Fe $\mathrm{II}], \mathrm{H}_{2}$, and $\mathrm{H}$ recombination lines), we first fitted the emission line profiles by a single Gaussian, using the PROFIT fitting routine (Riffel 2010). Figure 1 shows, in the top panels, the $[\mathrm{Fe}$ II] $\lambda 1.2570 \mu \mathrm{m}$ flux map in the left panel and the $\sigma$ map in the right panel. 

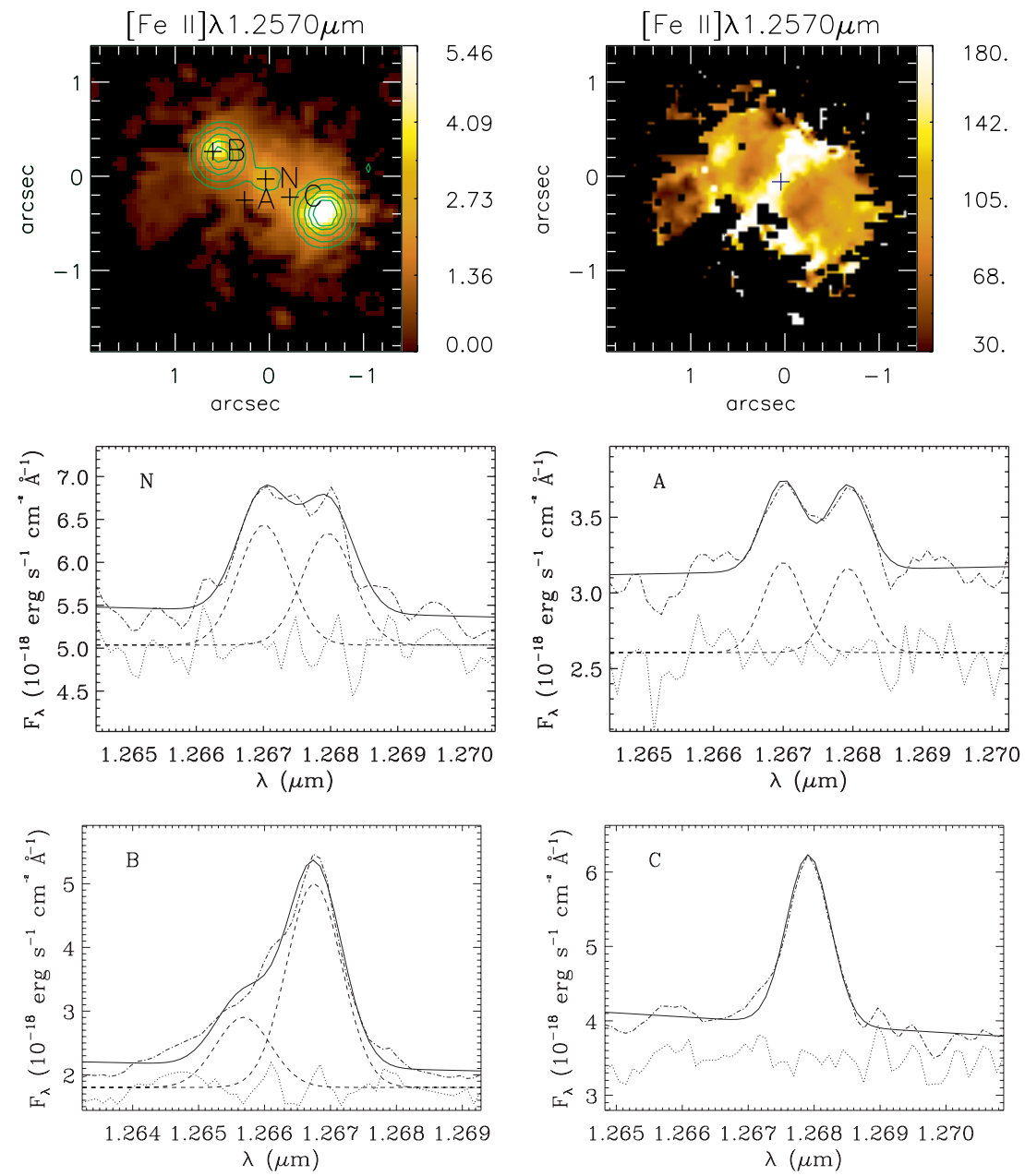

Figure 1. Top-left: [Fe II] $\lambda 1.25 \mu \mathrm{m}$ flux distribution with contours of the radio image overlaid (Ulvestad \& Wilson 1989). Top-right: $\sigma$ of the one-component fits to the lines, where the SE-NW strip can be seen at P.A. $=-30^{\circ}$. The remaining panels show examples of the fitting of the [Fe II] $\lambda 1.25 \mu \mathrm{m}$ emission line profile by two Gaussian curves (positions N, A, and B) and by a single Gaussian (position C). The spectra are shown as dashed-dotted lines, the resulting fit as solid lines, each Gaussian component (plus a constant) as dashed lines and the residuals of the fit (plus a constant) as dotted lines.

(A color version of this figure is available in the online journal.)

The [Fe II] velocity field obtained from the one-component fit is very similar to that of a previous study by Stoklasová et al. (2009) for optical emission lines and we thus do not show it here. It presents the highest blueshifts of $\sim-250 \mathrm{~km} \mathrm{~s}^{-1}$ to the north-east of the nucleus, while similar redshifts are seen to the south-west of it. The maximum velocity gradient is observed along P.A. $\approx 55^{\circ}$. Our data has also allowed us to obtain the stellar velocity field (R. A. Riffel et al., in preparation); we found it to be similar to that obtained in the optical (Stoklasová et al. 2009). It presents an amplitude of less than $100 \mathrm{~km} \mathrm{~s}^{-1}$, with the line of nodes oriented along P.A. $\approx 35^{\circ}$. But the redshifts and blueshifts are observed to the north-east and south-west, respectively, thus contrary to what is observed in the gas velocity field, indicating that the gas is in counter-rotation relative to the stars.

The $\sigma$ map obtained from the one-component fit (top right panel of Figure 1) shows that the width of the Gaussians in a strip oriented along position angle P.A. $=-30^{\circ}$ (and also in a few other locations close to the radio hot spots) is almost twice the width of the profiles at other locations. A close inspection of these profiles reveals that at the locations where the profile is broader, they are in fact better fitted by two components and we repeated the fit using two components where necessary.
The distinct profiles observed over the nuclear region and their fits are illustrated in the bottom panels of Figure 1. We find that, besides the positions $\mathrm{N}$ and $\mathrm{A}$, shown in Figure 1, all profiles along P.A. $=-30^{\circ}$, in a strip $\approx 50 \mathrm{pc}$ wide, crossing the nucleus perpendicularly to the radio jet, have two components. We hereafter call this region the SE-NW strip. Two components are also observed at the locations of the radio hot spots (e.g., position $\mathrm{B}$ ), while for the remaining regions the profile is well reproduced by a single Gaussian (e.g., position C) with a similar width to that of the individual components of the two-component profiles.

We now discuss the velocity fields derived for the different gas components. We found that the $\sigma$ of the Gaussians fitted to the regions where only one component is necessary is similar to that of each of the two components (where two components were clearly necessary), being in the range $70-100 \mathrm{~km} \mathrm{~s}^{-1}$. We fitted two Gaussians to the [Fe II] profile only in locations where the fit of one Gaussian resulted in $\sigma$ higher than $100 \mathrm{~km} \mathrm{~s}^{-1}$. In regions where the profiles have $\sigma<100 \mathrm{~km} \mathrm{~s}^{-1}$ one Gaussian reproduces well the observed profile (see panel C of Figure 1). Besides this criterion, if the two components present differences in velocity less than our spectral resolution $\left(\approx 40 \mathrm{~km} \mathrm{~s}^{-1}\right)$ and/or one component had a flux lower than $5 \%$ of the other, 

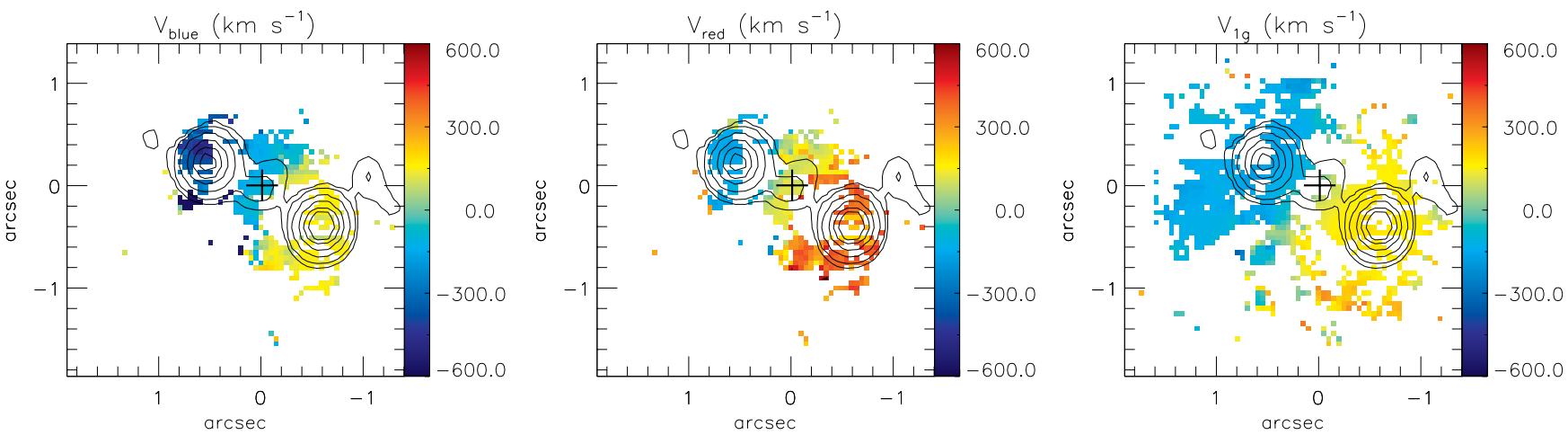

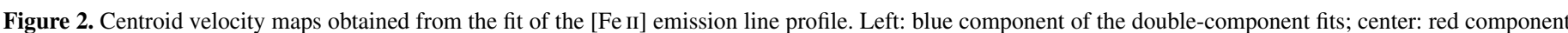

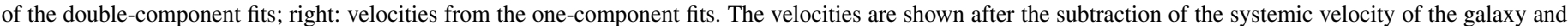
the color bars represent velocities in units of $\mathrm{km} \mathrm{s}^{-1}$.

(A color version of this figure is available in the online journal.)

we used the result of the fit of a single Gaussian. In addition, we kept the width of the two components the same (as this seems to work for the higher signal-to-noise regions), in order to have more robust fits, with fewer free parameters.

The right panel of Figure 2 shows the velocity field for the region where only one Gaussian was necessary to fit the [Fe II] profile, while the left and central panels show, respectively, the velocity field of the blue and red components of the two Gaussian fits. Besides the SE-NW strip, two components are also clearly present at and around the radio hot spots. It can be seen that the highest blueshifts — of up to $-600 \mathrm{~km} \mathrm{~s}^{-1}$ — correspond to the bluest components of the two-component fit in the region of the north-east hot spot, while the highest redshifts - of up to $600 \mathrm{~km} \mathrm{~s}^{-1}$ —are the reddest components of the two-component fit in the region of the south-east hot spot.

\section{DISCUSSION}

Optical IFS was obtained for the first time for NGC 5929 by Ferruit et al. (1997) at an angular resolution of $\approx 1$ arcsec and a spectral resolution of $R \sim 1400$. They indicate that the gas to the north-east is observed in blueshift and to the south-west in redshift, focusing on the apparent association between the gas emission and kinematics with the radio jet. They report the finding that the width of the [OIII] $\lambda 5007$ emission line at the nucleus is twice its width to the north-east and south-west, attributing this to the interaction with the radio jet, but their measurements did not allow resolving the extent of the region at the nucleus with high velocity dispersions, or to conclude that the emission lines actually had two velocity components.

Rosario et al. (2010) found kinematic shock signatures attributed to the interaction of the radio jet with the NLR gas in NGC 5929 using Hubble Space Telescope optical images, long-slit spectra, and a $5 \mathrm{GHz}$ radio image. They compared the emission line profiles of $\mathrm{H} \beta$ with those of $[\mathrm{O}$ III] $\lambda 5007$ at distinct positions in the galaxy and found that they have similar widths at most locations, except in a region surrounding the north-eastern radio hot spot. There, the $\mathrm{H} \beta$ profile is broader than that of [O III], suggesting that the low-ionization gas at the interaction site is more disturbed than the high-ionization gas. The authors attributed this broadening to a local enhancement of shock ionization due to the influence of the jet. The spectra shown by Rosario et al. (2010) do not include observations in the direction perpendicular to the radio jet, thus missing the SE-NW strip region, or a nuclear spectrum, and only show the emission line profiles at four distinct locations.
We can compare our results with those of Rosario et al. (2010) for the regions in common. Although the width of the $\mathrm{Pa} \beta$ profile around the position of the north-eastern radio hot spot is similar to those observed for $\mathrm{H}_{\beta}$, the $\mathrm{Pa} \beta$ profile does not present any hint of broadening or an additional component at this location relative to the surroundings. One possibility is that a second component is not detected at $\mathrm{Pa} \beta$ because it is not bright enough, even though it can be detected at $\mathrm{H}_{\beta}$, because $\mathrm{H}_{\beta}$ is 6.2 times stronger than $\mathrm{Pa} \beta$ (Osterbrock \& Ferland 2006). On the other hand, we observe two components in the [Fe II] profile at the locations of the radio hot spots. The [Fe II] traces even lower ionization gas than $\mathrm{Pa} \beta$ as the ion Fe II is formed in partially ionized regions, having an ionization potential of only $7.9 \mathrm{eV}$ (Worden et al. 1984), while the $\mathrm{Pa} \beta$ line originates from a fully ionized region (e.g., Storchi-Bergmann et al. 2009). Although many of our results in the near-infrared support those by Rosario et al. (2010), a new result we have obtained relative to their study is the observation of the double-component profiles along the SE-NW strip, extending by $\approx 300$ pc and running perpendicularly to the main radio jet. This structure was not observed by Rosario et al. (2010) due to their restricted spatial coverage.

Whittle et al. (1986) have suggested that the double components observed perpendicularly to the radio jet (which is approximately the orientation of the minor axis of the galaxy) could be interpreted as being originated in a rotating disk by the superposition of two components from opposite sides of the rotation curve which would have been unresolved by their observations. We note, however, that even at our improved spatial resolution of $\approx 20 \mathrm{pc}$, the emission line profiles of the lines along the SE-NW strip are still double. Moreover, they are double not only along the presumed rotation major axis, where a velocity gradient is expected, but also along the presumed kinematic minor axis, where the velocity of a rotation field should be zero. Our data thus do not support the hypothesis that the two components observed along the minor axis of the galaxy are due to unresolved rotation.

In order to better inspect the gas kinematics close to the nucleus, we show in Figure 3, a "three-dimensional (3D) rendering" of the velocity field: the spatial coordinates $x, y$ define the galaxy plane, and the centroid velocity is plotted as the third coordinate $z$. In this plot we show only the velocities corresponding to the two components along the SE-NW strip and those corresponding to the one-component fits (excluding the two components associated with the radio hot spots). The velocity field of the one-component region (in green) seems to 


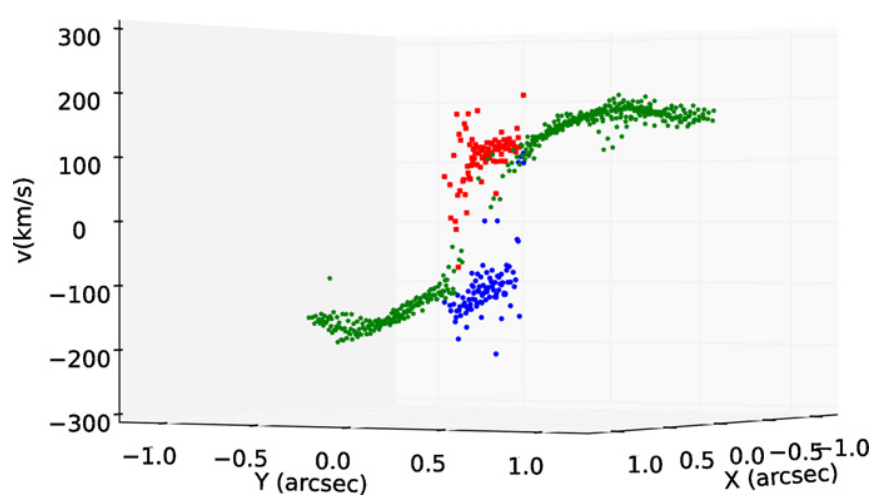

Figure 3. Velocity 3D plot: the blue component is shown in blue, the red component in red, and the single Gaussian component in green.

(A color version of this figure is available in the online journal.)

delineate a rotation curve, while the blueshifted and redshifted components (in blue and red, respectively) of the SE-NW strip seem to be detached form the one-component velocity field (even though the velocities are similar), indicating a distinct component.

We have fitted the one-component velocity field using a rotating disk model (e.g., Riffel et al. 2013). The measured velocity field, the best fit rotation model, and the residuals are shown in Figure 4. The best fit model shows quite a steep rotation curve, and the presence of a few high residuals show that the velocity field is only approximately fitted by rotation.

As the gas velocity field rotates in the opposite direction to that of the stars, our interpretation is that this gas was captured in the interaction with NGC 5930 and is now rotating around the nucleus of NGC 5929, but still showing distortions from a "regular" rotation field due to interaction with the companion. Inflow toward the center of this gas was probably the trigger of the nuclear activity in NGC 5929. The onset of nuclear activity is associated with outflows originating in the accretion disk, and if a strong "equatorial outflow" from the accretion disk could be generated, its interaction with the surrounding gas in the galaxy-pushing it outward - could be the origin of the double-component profiles seen along the SE-NW strip. The presence of such an outflow is nevertheless peculiar, as the best mapped outflows in nearby Seyfert galaxies are oriented approximately along the radio jet, not perpendicular to it (e.g., Storchi-Bergmann et al. 1992, 2010; Das et al. 2005, 2007).

"Equatorial" outflows from accretion disks have been recently proposed by Li et al. (2013), which are also compatible with a scenario in which the torus surrounding the active galactic nucleus is actually a dusty wind emanating from the outer parts of the accretion disk (e.g., Hönig et al. 2013; Elitzur 2012; Ivezić \& Elitzur 2010; Mor et al. 2009; Nenkova et al. 2008; Elitzur $\&$ Shlosman 2006). We conclude that the peculiar velocity field observed in the SE-NW strip is thus probably the result of the interaction of an accretion disk equatorial outflow or torus wind with the surrounding gas in the galaxy.

In R. A. Riffel et al. (in preparation) we find that the line ratios suggest a contribution of shocks to the excitation of the $[\mathrm{Fe} \mathrm{II}]$ and $\mathrm{H}_{2}$ lines along P.A. $=-30^{\circ}$, supporting the presence of an outflow there. In fact, Su et al. (1996) presented MERLIN and Very Large Array radio images for NGC 5929 at frequencies ranging from 15 to $0.4 \mathrm{GHz}$ and resolutions from 0.05 to 0.6 . The radio emission at all frequencies is dominated by the three components seen in the contours of Figure 1: the two hot spots plus a nuclear component. But the $0.4 \mathrm{GHz}$ image shows an additional extended emission oriented approximately perpendicular to the line connecting the two hot spots, along P.A. $\approx-30^{\circ}$-thus along the SE-NW strip. This extended radio emission has been attributed by $\mathrm{Su}$ et al. (1996) to a flow of relativistic particles generated by the active nucleus flowing out perpendicularly to the radio jet. A similar scenario was proposed by Pedlar et al. (1993) for NGC 4151. Su et al. (1996) also discuss an alternative scenario where the extended radio emission originates from starburst driven winds. This scenario can nevertheless be discarded by our observations since the [Fe II] $/ \mathrm{Pa} \beta$ and $\mathrm{H}_{2} / \mathrm{Br} \gamma$ ratios are higher than those expected for starbursts (R. A. Riffel et al., in preparation).

Finally, we have also found double components in the emission line profiles from regions surrounding the radio hot spots. We note that one of the components has approximately the velocity of the one-component gas in the surrounding regions, while the other component shows a higher velocity, of up to $\approx 600 \mathrm{~km} \mathrm{~s}^{-1}$ around the hot spots, in blueshift to the north-east and in redshift to the south-west. This indicates that the northeast side of the radio jet is tilted toward us. We support the interpretation of Rosario et al. (2010) that these high velocities are due to the radio jet interacting with the ambient medium, which in this case is the gas in counter-rotation, pushing it away from the galaxy nucleus.

\section{SUMMARY AND CONCLUSIONS}

We used near-infrared IFS of the inner 250 pc of NGC 5929 at a spatial resolution of $20 \mathrm{pc}$ and report the discovery of a $50 \mathrm{pc}$ wide strip crossing the nucleus perpendicularly to the radio jet
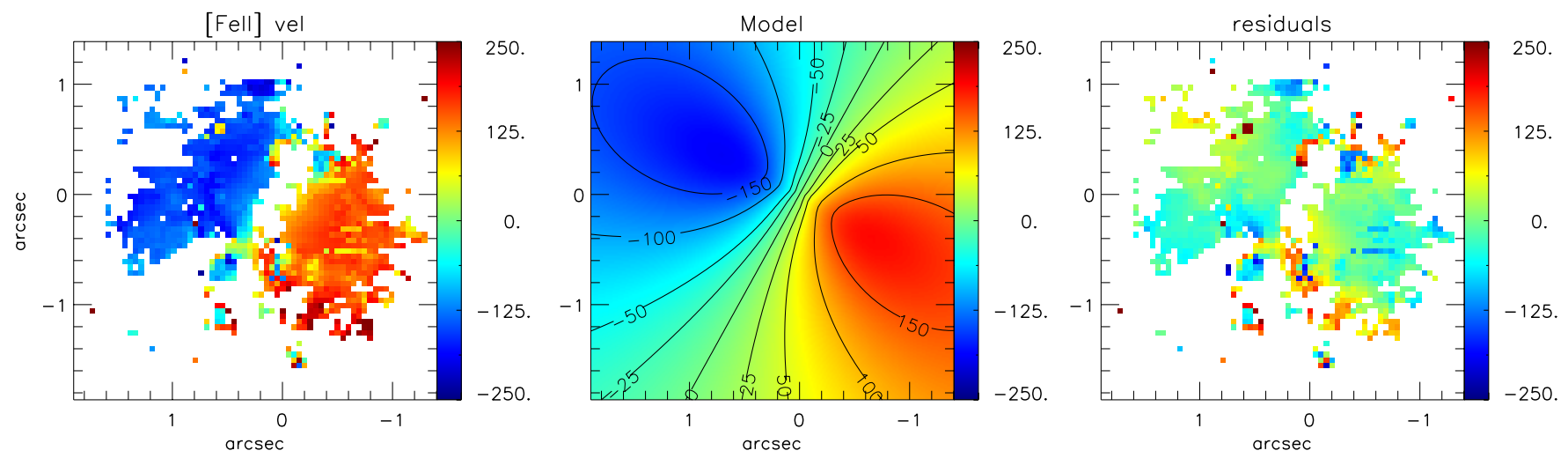

Figure 4. Left: [Fe II] velocity field attributed to rotation; center: rotating disk model; and right: residual map.

(A color version of this figure is available in the online journal.) 
(P.A. $=-30^{\circ}$, the SE-NW strip) and extending by $300 \mathrm{pc}$ where the emission lines show two velocity components, one at $\approx-150 \mathrm{~km} \mathrm{~s}^{-1}$ and the other at $\approx+150 \mathrm{~km} \mathrm{~s}^{-1}$.

We also show that the extended gas emission perpendicular to the SE-NW strip shows only one component, observed in blueshift to the north-east and redshift to the south-west, except around the region of the hot spots, where they are again double. This apparent rotation pattern is opposite to that of the stellar velocity field. We have fitted a rotation model to the onecomponent gas and found a steep rotation curve, and residuals as high as $100 \mathrm{~km} \mathrm{~s}^{-1}$, evidencing deviations from simple rotation, which we attribute to the effect of the companion galaxy NGC 5930.

A possible scenario for the complex gas velocity field we have observed is the following: the interaction with NGC 5930 has triggered the transfer of gas to the central region of NGC 5929. The observation that the gas is in counter-rotation relative to the stars supports an external origin. Part of this gas reaches the nucleus, where it triggers the nuclear activity, giving rise to the main radio jets which have the north-east side oriented toward us. The interaction of the radio jet with the rotating gas pushes the rotating gas to projected velocities along the line of sight of up to $600 \mathrm{~km} \mathrm{~s}^{-1}$.

The nuclear activity seems to also have produced equatorial outflows along the accretion disk, whose interaction with the surrounding gas-pushing it outward-originated the double components observed in the emission line profiles along the SE-NW strip. The presence of such outflows is also supported by the previous observation of faint radio emission perpendicular to the main radio jet. It is also interesting to note that other recent integral field unit studies of the central region of active galaxies by our group, such as in Couto et al. (2013), Schnorr Müller et al. (2013), and in D. Lena et al. (in preparation) have also revealed broadened or double emission line profiles perpendicular to the ionization axis, suggesting that outflows perpendicular to the main axis of the active nucleus may not be a rare phenomenon. These observations support recent models of equatorial accretion disk winds (Li et al. 2013) and outflowing torus models (Hönig et al. 2013; Elitzur 2012; Ivezić \& Elitzur 2010; Mor et al. 2009; Nenkova et al. 2008; Elitzur \& Shlosman 2006).

We thank the referee for valuable suggestions which helped improve the paper. This work is based on observations obtained at the Gemini Observatory, which is operated by the Association of Universities for Research in Astronomy, Inc., under a cooperative agreement with the NSF on behalf of the Gemini partnership: the National Science Foundation (United States), the Science and Technology Facilities Council (United Kingdom), the National Research Council (Canada), CONICYT (Chile), the Australian Research Council (Australia), Ministério da Ciência e Tecnologia (Brazil), and Ministerio de Ciencia, Tecnologia e Innovación Productiva (Argentina). This work has been partially supported by the Brazilian institutions CNPq and FAPERGS.

\section{REFERENCES}

Cecil, G., Dopita, M. A., Groves, B., et al. 2002, ApJ, 568, 627

Couto, G. S., Storchi-Bergmann, T., Axon, D. J., et al. 2013, MNRAS, 435, 2982

Das, V., Crenshaw, D. M., Hutchings, J. B., et al. 2005, AJ, 130, 945

Das, V., Crenshaw, D. M., \& Kraemer, S. B. 2007, ApJ, 656, 699

Elitzur, M. 2012, ApJL, 747, L33

Elitzur, M., \& Shlosman, I. 2006, ApJL, 648, L101

Falcke, H., Wilson, A. S., \& Simpson, C. 1998, ApJ, 502, 199

Ferruit, P., Pecontal, E., Wilson, A. S., Binette, L., \& Wilson, A. S. 1997, A\&A, 328, 493

Ferruit, P., Wilson, A. S., Whittle, M., et al. 1999, ApJ, 523, 147

Hönig, S. F., Kishimoto, M., Tristram, K. R. W., et al. 2013, MNRAS, 771,87

Ivezić, Z., \& Elitzur, M. 2010, MNRAS, 404, 1415

Keel, W. C. 1985, Natur, 318, 43

Li, J., Ostriker, J., \& Sunyaev, R. 2013, ApJ, 767, 105

McGregor, P. J., Hart, J., Conroy, P. G., et al. 2003, Proc. SPIE, 4841, 1581

Mor, R., Netzer, H., \& Elitzur, M. 2009, ApJ, 705, 298

Nenkova, M., Sirocky, M., Ivezić, Z., \& Elitzur, M. 2008, ApJ, 608, 147

Osterbrock, D. E., \& Ferland, G. J. 2006, Astrophysics of Gaseous Nebulae and Active Galactic Nuclei (Mill Valley, CA: University Science Books)

Pedlar, A., Kukula, M. J., Longley, D. P. T., et al. 1993, MNRAS, 263, 471

Riffel, R. A. 2010, Ap\&SS, 327, 239

Riffel, R. A., \& Storchi-Bergmann, T. 2011, MNRAS, 411, 469

Riffel, R. A., Storchi-Bergmann, T., \& Winge, C. 2013, MNRAS, 430, 2249

Riffel, R. A., Storchi-Bergmann, T., Winge, C., \& Barbosa, F. K. B. 2006, MNRAS, 373, 2

Rosario, D. J., Whittle, M., Nelson, C. H., \& Wilson, A. S. 2010, ApJL, 711, L94

Schnorr Müller, A., Storchi-Bergmann, T., Nagar, N. M., et al. 2013, MNRAS, in press (arXiv:1310.7916)

Stoklasová, I., Ferruit, P., Emsellem, E., et al. 2009, A\&A, 500, 1287

Storchi-Bergmann, T., Lopes, R. D. S., McGregor, P. J., et al. 2010, MNRAS, 402, 819

Storchi-Bergmann, T., McGregor, P. J., Riffel, R. A., et al. 2009, MNRAS, 394, 1148

Storchi-Bergmann, T., Wilson, A. S., \& Baldwin, J. A. 1992, ApJ, 396, 45

Su, B. M., Muxlow, T. W. B., Pedlar, A., et al. 1996, MNRAS, 279, 111

Ulvestad, J. S., \& Wilson, A. S. 1984, ApJ, 285, 439

Ulvestad, J. S., \& Wilson, A. S. 1989, ApJ, 343, 659

Whittle, M., Haniff, C. A., Ward, M. J., et al. 1986, MNRAS, 222, 189

Whittle, M., \& Wilson, A. S. 2004, AJ, 127, 606

Wilson, A. S., \& Keel, W. C. 1989, AJ, 98, 1581

Worden, E. F., Comaskey, B., Densberger, J., et al. 1984, JOSAB, 1, 314 